\title{
Increased circulating CRTH2 + Tregs are associated with asthma control and exacerbation
}

Tadech Boonpiyathad ${ }^{1}$, Teerapol Chantveerawong ${ }^{1}$, Sasipa Sangkanjanavanich ${ }^{1}$, Chirawat Chiewchalermsri $^{1}$, Panitan Pradubpongsa ${ }^{1}$, Wat Mitthamsiri ${ }^{1}$, Sarawut Jindarat ${ }^{2}$, Ming Wang $^{3}$, Mübeccel Akdis ${ }^{4}$, Milena Sokolowska ${ }^{4}$, Cezmi Akdis ${ }^{4}$, and Atik Sangasapaviliya ${ }^{1}$

${ }^{1}$ Phramongkutklao Hospital

${ }^{2}$ Phramongkutklao College of Medicine

${ }^{3}$ Beijing Tongren Hospital

${ }^{4}$ Universitat Zurich Schweizerisches Institut fur Allergie- und Asthmaforschung

July 17, 2021

Increased circulating CRTH2 ${ }^{+}$Tregs are associated with asthma control and exacerbation

Short running title:Increased circulating $\mathrm{CRTH} 2^{+}$Tregs among patients with asthma

Teerapol Chantveerawong ${ }^{\mathrm{a}}$, Sasipa Sangkangjanavanich ${ }^{\mathrm{a}, \mathrm{b}}$, Chirawat Chiewchalermsri ${ }^{\mathrm{a}, \mathrm{c}}$, Panitan Pradubpongsa $^{\mathrm{a}}$, Wat Mitthamsiri ${ }^{\mathrm{a}}$, Sarawut Jindarat ${ }^{\mathrm{d}}$, Ming Wang ${ }^{\mathrm{e}}$, Mübeccel Akdis ${ }^{\mathrm{f}}$, Milena Sokolowska ${ }^{\mathrm{f}}$, Cezmi A. Akdis ${ }^{f}$, Atik Sangasapaviliya ${ }^{a}$, Tadech Boonpiyathad ${ }^{\mathrm{a}, \mathrm{f}}$

${ }^{a}$ Division of Allergy and Clinical Immunology, Department of Medicine, Phramongkutklao Hospital, Bangkok, Thailand

${ }^{\mathrm{b}}$ Division of Allergy, Immunology and Rheumatology, Department of Medicine, Faculty of Medicine Ramathibodi Hospital, Mahidol University, Bangkok, Thailand

${ }^{\mathrm{c}}$ Department of Medicine, Panyananthaphikkhu Chonprathan Medical Center, Srinakharinwirot University, Nonthaburi, Thailand

${ }^{\mathrm{d}}$ Department of Pharmacology, Phramongkutklao College of Medicine, Bangkok, Thailand

e Department of Otolaryngology, Head and Neck Surgery, Beijing TongRen Hospital, Capital Medical University, and the Beijing Key Laboratory of Nasal Diseases, Beijing Institute of Otolaryngology, Beijing, China

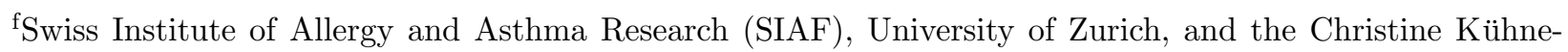
Center for Allergy Research and Education (CK-CARE), Davos, Switzerland

\section{Corresponding author:}

Tadech Boonpiyathad Division of Allergy and Clinical Immunology, Department of Medicine, Phramongkutklao Hospital, Bangkok, Thailand.

tadechb@pmk.ac.th

Tel: +66-2354-7614

Fax: +66-2763-3278

\section{KEYSWORDS}


Allergic, ALI culture, asthma, asthma control test score, asthma exacerbation, CRTH2, epithelial tight junction, PGD2, Treg cells, Tregs dysfunction

\section{Abbreviations}

ALI: Air-liquid interface

ACT: Asthma control test

CRTH2: Chemoattractant receptor-homologous molecule expressed on Th2 cells

HBEC: Human bronchial epithelial cell

PEFR: Peak expiratory flow rate

PGD2: Prostaglandin D2

TER: Transepithelial electrical resistance

Th2: T helper 2 cells

TJ: Tight junction

Tregs: Regulatory T cells

tSNE: t-Distributed stochastic neighbor embedding

\section{To the editor,}

Asthma is a chronic airway inflammatory disease consisting of several variable clinical presentations (phenotypes) and distinct pathophysiological mechanisms (endotypes). ${ }^{1}$ Chemoattractant receptor-homologous molecule expressed on Th2 cells (CRTH2) is a type 2 receptor for prostaglandin D2 (PGD2), expressed on Th2 and other various cell types including regulatory T cells (Tregs). ${ }^{2}$ Tregs can suppress proliferation and activation of effector type 2 inflammatory cells. On the other hand, dysregulated Tregs have been implicated in developing chronic allergic diseases. ${ }^{3}$ Among patients with allergic asthma, theFOXP3 gene expression and Treg suppressive function decrease compared with healthy controls. ${ }^{4}$ GATA-3 expression in Tregs may contribute to their Th2-like responses by switching Tregs to Th2 cells under the type 2 milieu. ${ }^{5}$ Dysregulated Tregs represent a Th2-like phenotype. That may be due to overexpression of GATA3 and producing high amounts of interleukin (IL)-4 after local exposure to PGD2. ${ }^{6}$ In this present study, we assessed the presence of $\mathrm{CRTH}_{2}{ }^{+}$Tregs in peripheral blood of patients with different inflammatory phenotypes of asthma. We found that the frequency of $\mathrm{CRTH}_{2}{ }^{+}$Tregs was increased in patients with asthma, particularly in those with concomitant allergy and this increase correlated negatively with asthma control. Furthermore, we discovered that the frequency of $\mathrm{CRTH} 2^{+}$Tregs increased during an acute asthma exacerbation. Finally, we demonstrated that Tregs of allergic patients with asthma exhibited Th2-biased character.

We first studied the frequency of $\mathrm{CRTH}_{2}^{+}$and CRTH2 ${ }^{-}$Tregs $\left(\mathrm{CD} 3^{+} \mathrm{CD} 4^{+} \mathrm{CD} 25^{+} \mathrm{CD} 127^{-}\right)$and $\mathrm{Th} 2$ $\left(\mathrm{CD}^{+}{ }^{\mathrm{CD}} 4^{+} \mathrm{CD} 25^{-} \mathrm{CRTH} 2^{+}\right)$in 120 patients with asthma compared with 30 healthy controls. For a detailed description of the method, see the methods section in this article's supplementary data. The gating strategy is shown inFig S1A. The frequency of CRTH2 ${ }^{+}$Tregs in the asthma group, 0.09\% [0.06-0.18], was significantly higher as compared to the control group, $0.04 \%[0.03-0.06], p<0.0001$ (Fig 1A ). The frequencies of CRTH2-Tregs in patients with asthma were significantly lower (Fig 1A ), whereas Th2 cells did not differ between controls and asthmatics (Fig S2A ). Next, we compared the frequency of T cell subsets in patients with different asthma phenotypes: eosinophilic allergic (EA), eosinophilic non-allergic (EN), non-eosinophilic allergic (NA) and non-eosinophilic non-allergic (NN)) patients and controls. The median of $\mathrm{CRTH}_{2}{ }^{+}$Tregs in EA, NA, and NN was significantly higher than in controls (Fig 1B and S1B ). No significant difference was observed in the median of CRTH2-Tregs and Th2 cells between asthma phenotypes and control (Fig S2B ). Patients with allergic asthma had higher frequencies of CRTH2 ${ }^{+}$Tregs than those with non-allergic asthma (Fig 1C ). Also, patients with controlled asthma exhibited lower $\mathrm{CRTH}_{2}^{+}$Tregs than those with uncontrolled asthma (Fig 1C ). In this study, well-controlled asthma was determined by a high 
asthma control test (ACT) score which correlated with a low number of CRTH2 ${ }^{+}$Tregs (Fig 1D ). Besides, percentage of predicted peak expiratory flow rate (PEFR) negatively correlated with $\mathrm{CRTH}^{+}$Tregs levels (Fig 1D ). We also observed that the frequency of CRTH2 ${ }^{+}$Tregs was associated with absolute eosinophil counts and Th2 cells (Fig S2C ).

To explore the role of $\mathrm{CRTH}_{2}{ }^{+}$Tregs in an asthma attack, we examined 30 asthma donors during exacerbation in the absence of infection and 15 controls (Fig S3A). The level of CRTH2 ${ }^{+}$Tregs was significantly higher among the patients with asthma exacerbation, $0.13 \%$ [0.1-0.22] than that of patients with controlled asthma, 0.09\% [0.08-0.11], $p=0.007$ (Fig 1E and S3B ). After three weeks of follow-up, the frequency of $\mathrm{CRTH} 2^{+}$Tregs in those patients during asthma control decreased significantly compared with periodic asthma exacerbation, median difference $0.03 \%, p=0.01$ (Fig 1E ), whereas the differences were not significant among patients with stable, controlled asthma.

Next, we analyzed the heterogeneity of T-cell phenotypes using t-distributed stochastic neighbor embedding (tSNE) algorithm. T cell populations could be classified based on clustering results, except CRTH2 ${ }^{+}$Tregs which grouped with Th2 cells (Fig 2A ). Of note, the numbers of $\mathrm{CRTH} 2^{+}$Tregs were higher among patients with allergic asthma than those with non-allergic phenotypes and healthy individuals. To examine the relative expression of Th2 genes in Tregs, we determined mRNA levels from purified Tregs of patients with asthma and healthy controls. We observed statistically higher expression of PTGDR2 mRNA in patients with allergic phenotype compared with those with the non-allergic phenotypes and healthy controls (Fig 2B ). Patients with asthma showed also significantly reduced FOXP3 and a trend to higher expression of GATA3 compared with controls (Fig 2C ). Accordingly, we observed higher expression of $I L 4$ and IL13 in Tregs of patients with allergic asthma as compared to healthy controls, but IL10 showed no significant difference between the groups (Fig 2D ). These data indicate that type-2 gene expression was elevated, whereas regulatory signature was decreased in patients with asthma, particularly in those with allergic phenotypes.

All patients during asthma exacerbation received systemic corticosteroid treatment. We hypothesized that corticosteroids might affect the frequency of the CRTH2 ${ }^{+}$Tregs. To assess the efficacy of dexamethasone and DP2 antagonist (ramatroban) in response to PGD2 engagement, we examined activated (CD69 ${ }^{+}$) $\mathrm{CRTH}_{2}{ }^{+}$Tregs. Reduced CD69 expression was observed in the presence of dexamethasone with and without PGD2 compared with only PGD2 stimulation (Fig 2E and S4A ). Under DP2 antagonist treatment, the frequency of activated $\mathrm{CRTH}_{2}{ }^{+}$Tregs decreased compared with the condition with only PGD2 stimulation (Fig 2F and S4B ). These data indicate that dexamethasone and DP2 antagonists could alleviate CRTH2 ${ }^{+}$Tregs activation.

Next, we determined whether CRTH2 ${ }^{+}$Tregs might influence the bronchial epithelial barrier. After the first hour of the coculture, transepithelial electrical resistance (TER) was significantly lower in the presence of $\mathrm{CRTH}_{2}{ }^{+}$Tregs with PGD2 than CRTH2 ${ }^{-}$Tregs (Fig 2G ). Noticeably, TER in the presence of CRTH2 ${ }^{+}$Tregs with or without PGD2 significantly decreased after 6, 24, 48, and 72 hours compared with the control values (Fig 2G ). As shown in Fig S5, TJ protein was markedly disrupted in the HBEC-CRTH2 ${ }^{+}$Tregs cocultures compared with the absence of CRTH2 ${ }^{+}$Tregs. In this study, CRTH2 ${ }^{+}$Tregs stimulated with $\mathrm{PGD}_{2}$ led to slightly increased levels of IL-4, IL-5 and IL-13 compared with CRTH2 ${ }^{+}$Tregs without of PGD, but without a statistically significant difference between both groups (Fig $\mathbf{2 H})$. Moreover, type 2 cytokines were produced by $\mathrm{CRTH}_{2}{ }^{+}$Tregs more than CRTH2-Tregs (Fig $\mathbf{2 H}$ ).

In the current study, we investigated CRTH2 ${ }^{+}$Tregs in a sufficient sample size. However, the main limitation though is the lack of the broader and very detailed investigation of the differences between $\mathrm{CRTH} 2^{+}$Tregs, CRTH2-Tregs and Th2 cells. Further studies will determine other surface markers, transcription factors and cytokines which may lead to deeper understanding of the importance of these populations in the steady state and in allergic disease.

Here, we suggested that CRTH2 $2^{+}$Tregs might be implicated in the immunopathology of allergic asthma. However, CRTH2 ${ }^{+}$Tregs are not very frequent and the suppressive effect of CRTH2-Tregs may overwhelm these dysfunctional Tregs. Currently, many novel antagonists for PGD2 receptors have entered clinical trials. 
Information whether CRTH2 ${ }^{+}$Tregs might be a target for this new class of drugs seems to be of particular interest. Additional validation of the $\mathrm{CRTH} 2^{+}$Tregs approach as a biomarker will be needed to support clinical implementations.

\section{ACKNOWLEDGMENTS}

This study was supported by Phramongkutklao Hospital and Phramongkutklao College of Medicine foundation Scholarship and Christine Kühne-Center for Allergy Research and Education (CK-CARE), Davos. The authors wish to acknowledge Mrs. Jitta Tubkate for help in contacting the patients and Asst.Prof. Nattapon Jaisupa for advice in the laboratory work. We thank Dr. David Groeger for proofreading our manuscript.

\section{Conflict of Interest}

The authors do not have any conflicts of interest in relation to this work.

\section{Authors' Contribution}

T.C., S.S., C.C., and T.B. carried out the experiment. T.B., S.J., M.A., M.S, and C.A. conceived and planned the experiments. T.B. and M.S. wrote the manuscript with support from A.S., M.A. and C.A. T.B., T.C., S.S, C.C, P.P. and W.M. recruited the participants. T.C., S.S., C.C. and T.B. prepared the samples. M.W. prepared the HBEC samples and ALI culture. T.B., A.S. and C.A. supervised the study.

\section{REFERENCES}

1. Diamant Z, Vijverberg S, Alving K, et al. Toward clinically applicable biomarkers for asthma: An EAACI position paper.Allergy. 2019;74(10):1835-1851.

2. Boonpiyathad T, Sozener ZC, Akdis M, Akdis CA. The role of Treg cell subsets in allergic disease. Asian Pac J Allergy Immunol.2020;38(3):139-149.

3. Boonpiyathad T, Sozener ZC, Satitsuksanoa P, Akdis CA. Immunologic mechanisms in asthma. Semin Immunol. 2019;46:101333.

4. Marques CR, Costa RS, Costa GNO, et al. Genetic and epigenetic studies of FOXP3 in asthma and allergy. Asthma Res Pract.2015;1:10.

5. Kim BS, Kim IK, Park YJ, et al. Conversion of Th2 memory cells into Foxp3+ regulatory T cells suppressing Th2-mediated allergic asthma.Proc Natl Acad Sci U S A. 2010;107(19):8742-8747.

6. Boonpiyathad T, Capova G, Duchna HW, et al. Impact of high-altitude therapy on type-2 immune responses in asthma patients. Allergy.2020;75(1):84-94.

\section{Figure legend}

Figure 1. A , Frequencies of $\mathrm{CRTH}_{2}{ }^{+}$and $\mathrm{CRTH} 2^{-}$Tregs in patients with asthma $(\mathrm{n}=120)$ and healthy controls $(\mathrm{n}=30)$. B , Frequency of $\mathrm{CRTH}_{2}{ }^{+}$Tregs in patients with eosinophilic allergic $(\mathrm{EA}, \mathrm{n}=30)$, eosinophilic non-allergic $(\mathrm{EN}, \mathrm{n}=7)$, non-eosinophilic allergic $(\mathrm{NA}, \mathrm{n}=69)$, non-eosinophilic non-allergic $(\mathrm{NN}, \mathrm{n}=$ 14) and healthy control $(\mathrm{HC}, \mathrm{n}=30)$. $\mathbf{C}$, Frequency of $\mathrm{CRTH} 2^{+}$Tregs compared between allergic and uncontrolled asthma (asthma control test $(\mathrm{ACT})<20$ ). D , Correlation between the frequency of CRTH2 ${ }^{+}$Tregs and ACT and \% predicted peak expiratory flow rate (PEFR) in all asthmatic patients. E, Frequency of $\mathrm{CRTH}_{2}{ }^{+}$Tregs in patients with asthma exacerbation $(\mathrm{n}=30)$ and controlled $(\mathrm{n}=15)$ compared between baseline and after 3 weeks. ${ }^{*} p<0.05,{ }^{* *} p<0.01,{ }^{* * * *} p<0.0001$.

Figure 2. A,Representative tSNE plot clustered $6 \mathrm{~T}$ cells population, $\mathrm{CRTH} 2^{+} \mathrm{CD} 4{ }^{+} \mathrm{CD} 25^{-}$(dark blue), $\mathrm{CRTH}_{2}{ }^{\mathrm{CD}} 8^{+}$(dark red), $\mathrm{CRTH} 2^{+}$Tregs (dark green), $\mathrm{CRTH}^{-} \mathrm{CD} 4^{+} \mathrm{CD} 25^{-}$(light blue), $\mathrm{CRTH} 2^{-} \mathrm{CD} 8^{+}$ (light red) and CRTH2-Tregs (light green) of patient with allergic, non-allergic asthma and healthy control. RelativePTGDR2 (B), transcription factor genes(FOXP3 andGATA3 ) (C), cytokine genes (IL10, IL4 and $I L 13)(\mathbf{D})$ expression in Tregs of patients with allergic $(\mathrm{n}=10)$, non-allergic asthma $(\mathrm{n}=10)$ and healthy controls $(\mathrm{n}=10) . \mathbf{E}$, In vitro experiment, after 3 days of $\mathrm{CD}^{+} \mathrm{T}$ cells culture, $10^{-5} \mathrm{M}$ dexamethasone (Dexa) 
decreased CD69 expression on CRTH2 ${ }^{+}$Tregs in condition with/without PGD2 stimulation of asthmatic patients $(\mathrm{n}=10) . \mathbf{F}$, Fresh whole blood of asthmatic patients $(\mathrm{n}=10)$ were pretreated with $1 \mu \mathrm{M}$ ramatoban (DP2 antagonist) and then stimulated with PGD2. The numbers of CD69+ ${ }^{+} \mathrm{CRTH} 2^{+}$Tregs were investigated 1 hour after PGD2 administration. G , Tight junction of bronchial epithelial cells of asthmatic patients (n = 10) compared between unstimulated, PGD2, CRTH2 ${ }^{+}$Tregs with or without PGD2 and CRTH2-Tregs. Transepithelial electrical resistance (TER) measurements were examined at $0,1,6,24,48$, and 72 hours.H , Levels of IL-4, IL-5 and IL-13 in the supernatant of bronchial epithelial culture. ${ }^{*} p<0.05,{ }^{* *} p<0.01,{ }^{* * *} p$ $<0.001,{ }^{* * * *} p<0.0001$.

A
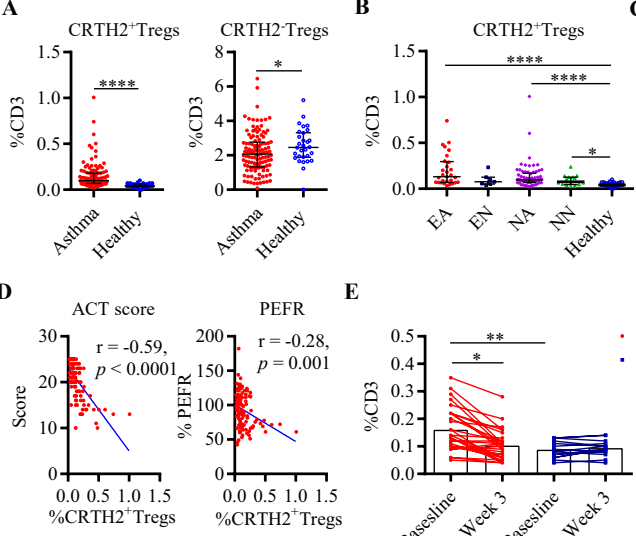

E
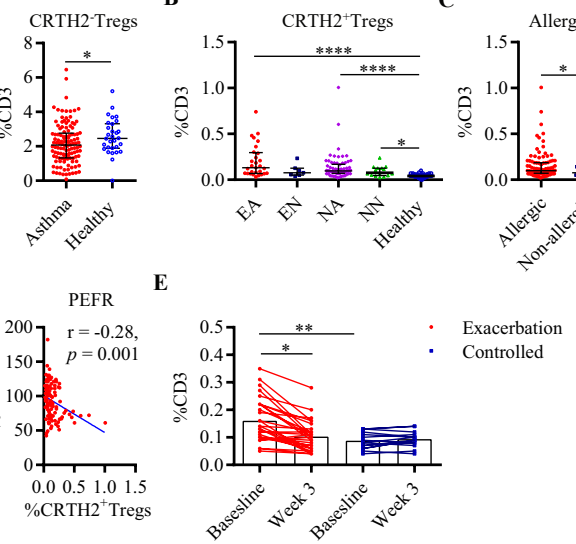

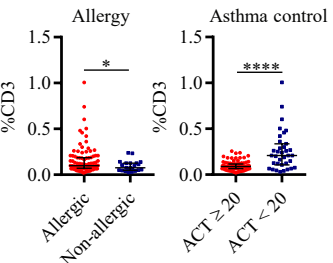

Figure 1. Chantveerawong et al. 
A
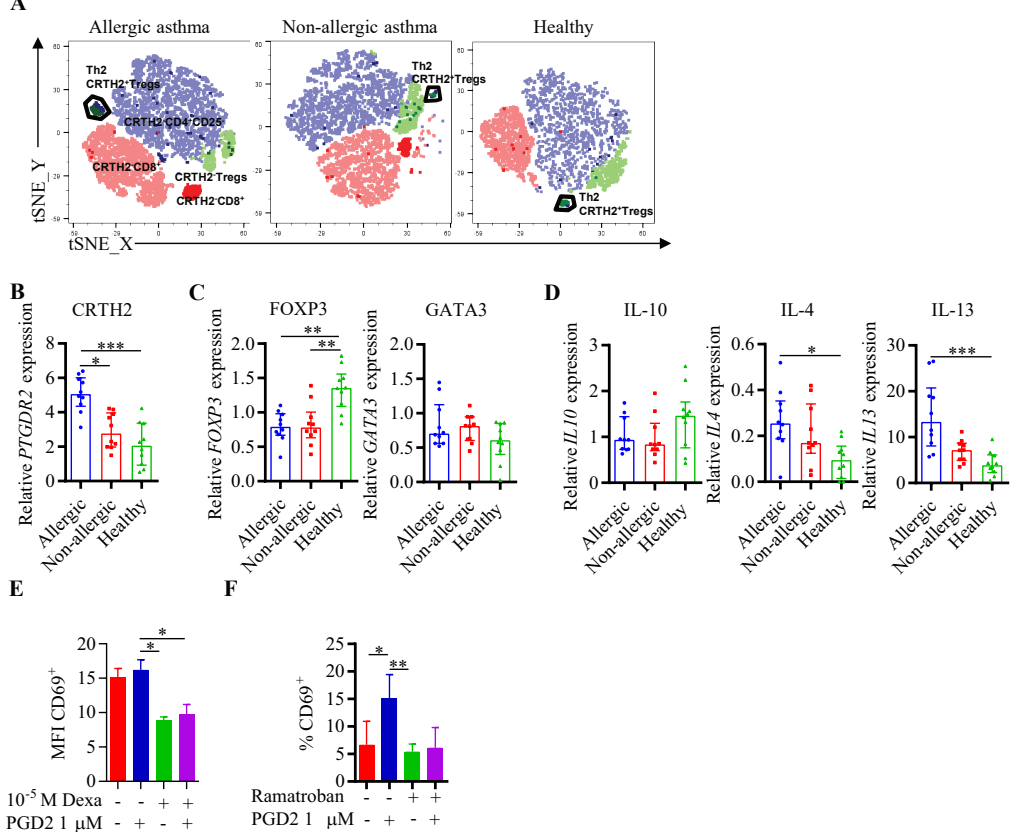

$\mathrm{PGD} 21 \mu \mathrm{M}-+\ldots$

G

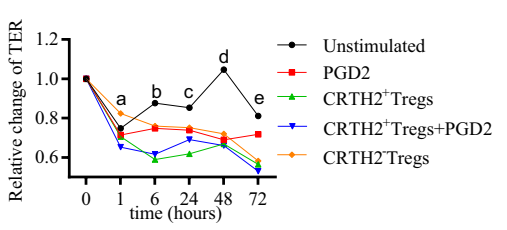

a CRTH2 ${ }^{2}$ Tregs+PGD2 vs. CRTH2-Tregs *

Unstimulated vs. CRTH2 ${ }^{2}$ Tregs + PGD2 $2 * * *$

CRTH2'Tregs vs. CRTH2-Tregs

CRTH2 ${ }^{+}$Tregs + PGD2 vs. CRTH2-Tregs *

c Unstimulated vs. CRTH2 Tregs ***

Unstimulated vs. CRTH2 $2^{+}$Tregs + PGD2

Unstimulated vs. CRTH2 ${ }^{2}$ Tregs + PGD2 $* * * *$

Unstimulated vs. CRTH2-Tregs ****

Unstimulated vs. CRTH2 ${ }^{2}$ Tregs + PGD2 $* * * *$

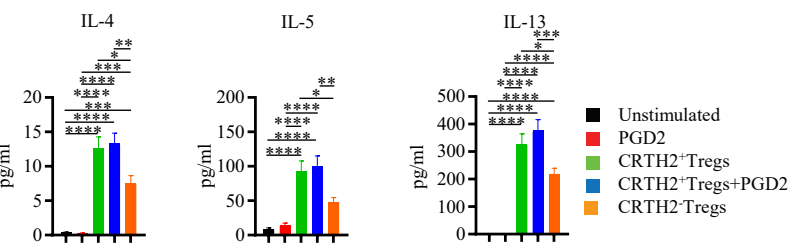

Figure 2. Chantveerawong et al. 

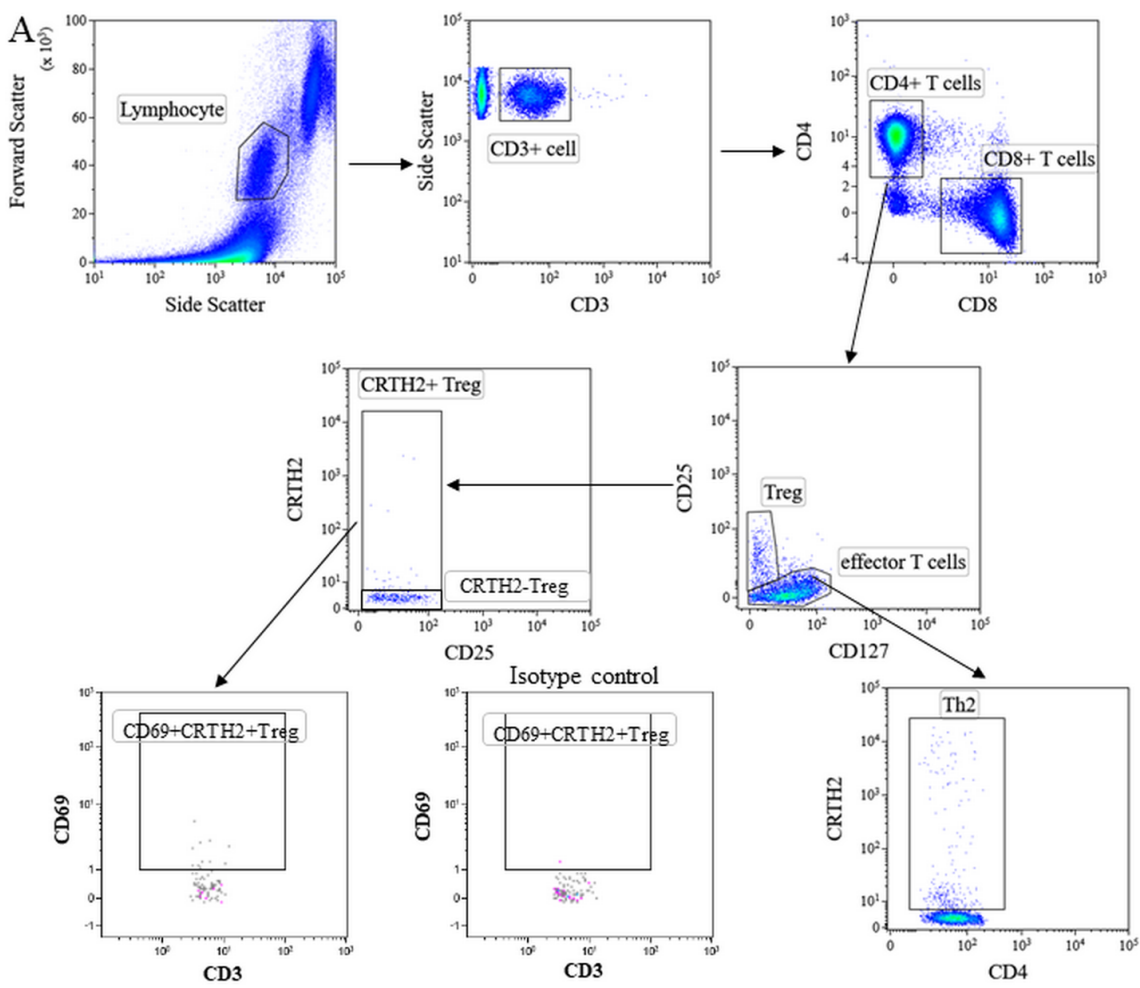

B

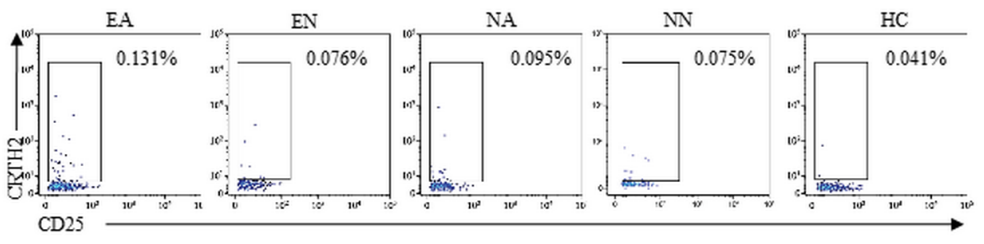

Figure S1. Chantveerawong et al. 


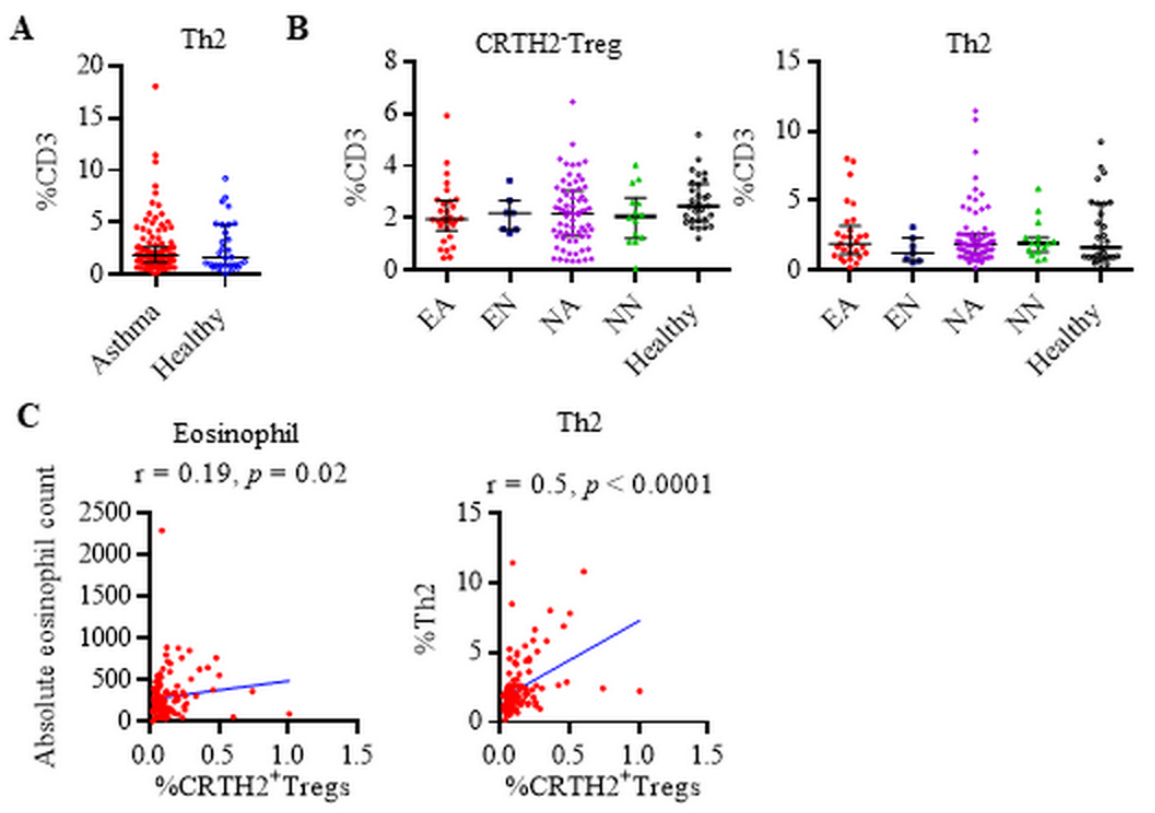

Figure S2. Chantveerawong et al. 

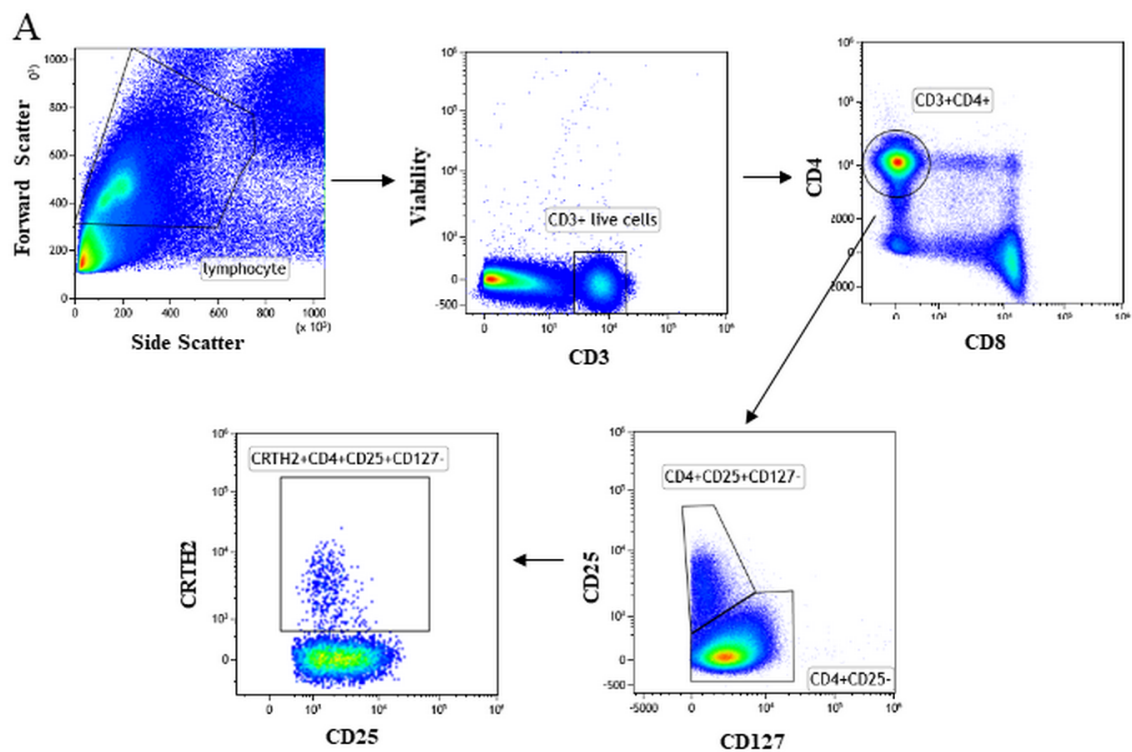

B Asthma exacerbation Asthma control

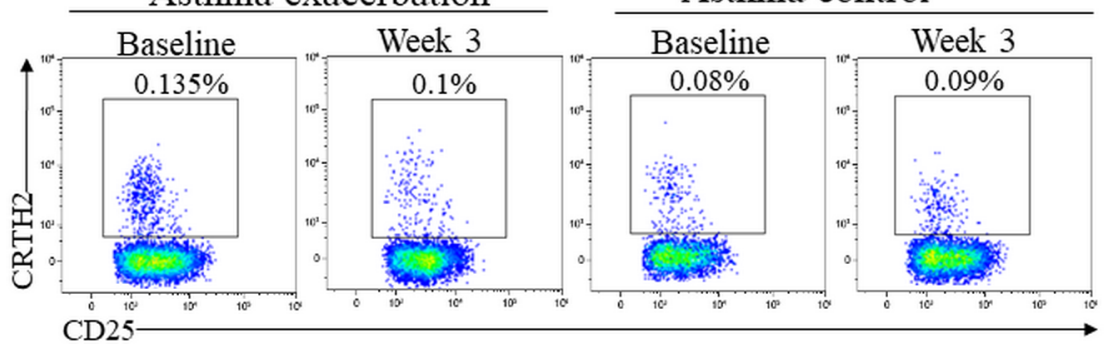

Figure S3 Chantveerawong et al. 


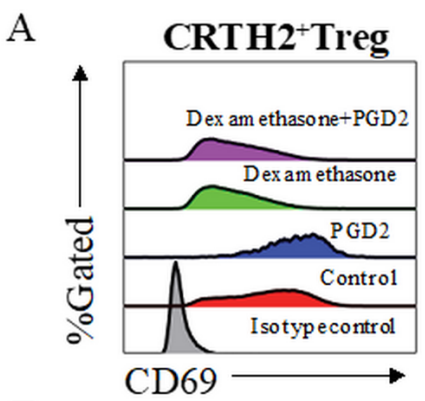

B
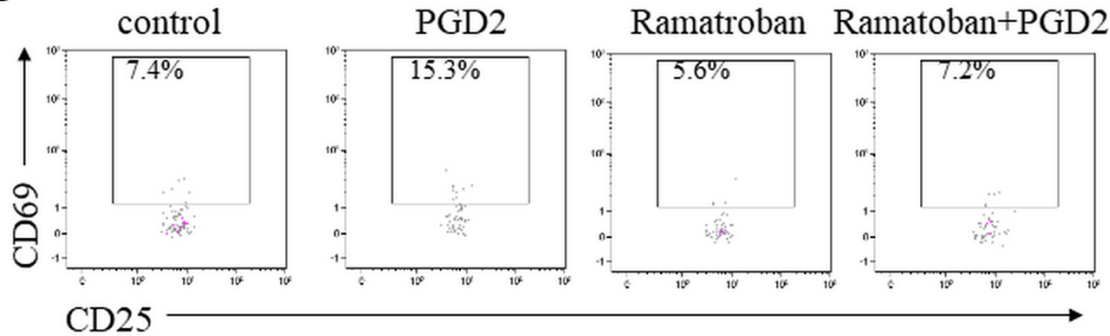

CD25

Figure S4 Chantveerawong et al.
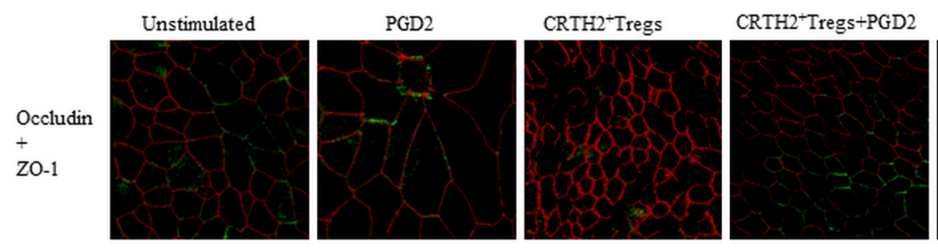

CRTH2-Tregs

Figure S5 Chantveerawong et al. 
A
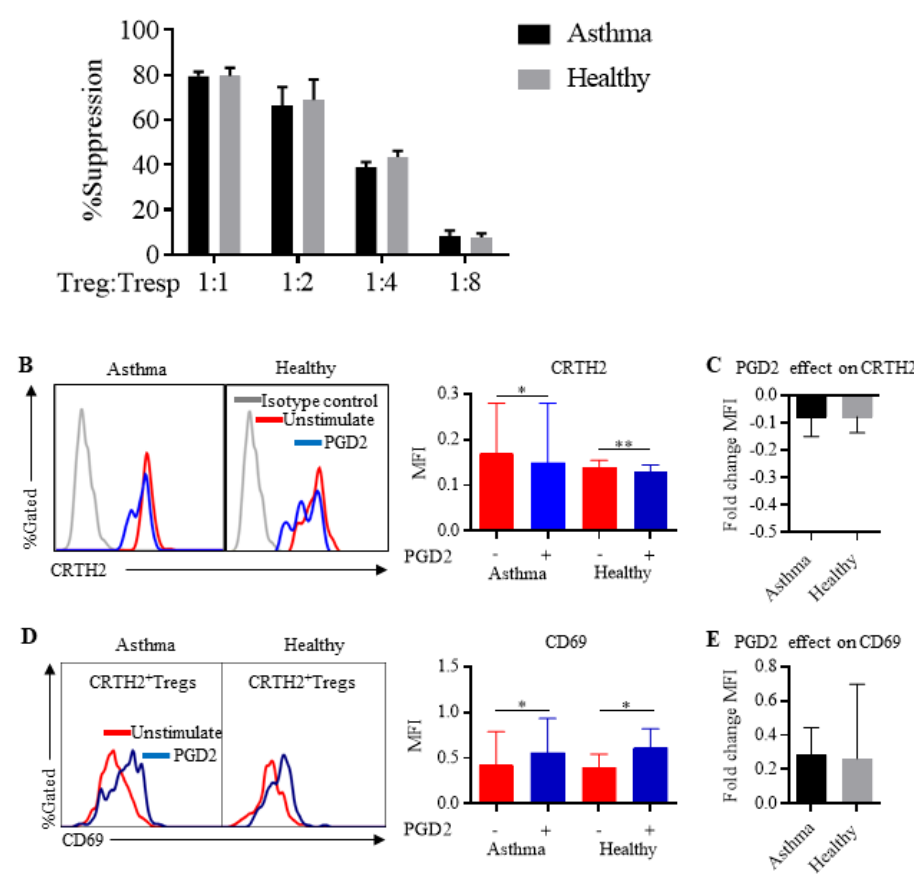

Figure S6 Chantveerawong et al. 